\title{
The ATLAS Trigger System: Recent Experience and Future Plans
}

\author{
Sinead FARRINGTON for the ATLAS Collaboration * \\ University of Oxford \\ E-mail: s.farrington1@physics.ox.ac.uk
}

The ATLAS detector at the LHC will experience unprecedented rates of hadron hadron collisions. The trigger system needs to reject efficiently a large rate of background events and still select potentially interesting events with high efficiency. After a first level trigger implemented in custom electronics, the trigger event selection is made by the High Level Trigger (HLT) system, implemented in software. To reduce the processing time to manageable levels, the HLT uses seeded, step-wise and fast selection algorithms, aiming at the earliest possible rejection of background events. The ATLAS trigger event selection is based on the reconstruction of potentially interesting physical objects like electrons, muons, jets, etc. The recent LHC startup and short single-beam run provided the first test of the trigger system against real data. Following this period, ATLAS continued to collect cosmic-ray events for detector alignment and calibration purposes. Both running periods provided very important data to commission the trigger reconstruction and selection algorithms. Profiting from this experience and taking into account the ATLAS first year physics goals, we are preparing a trigger selection menu including several tracking, muon-finding and calorimetry algorithms. Using Monte Carlo simulated data, we are evaluating the impact of the trigger menu on physics performance and rate.

European Physical Society Europhysics Conference on High Energy Physics

July 16-22, 2009

Krakow, Poland

* Speaker.

${ }^{\dagger}$ A footnote may follow. 


\section{Introduction}

The LHC will collide hadrons at an unprecedented rate. Beam bunches colliding at a rate of $40 \mathrm{MHz}$ lead to on average 23 collisions per crossing at the design luminosity. This gives an interaction rate of $1 \mathrm{GHz}$. The challenge to the ATLAS trigger system is to select the events which are of interest and record them on disk at the rate of $100-200 \mathrm{~Hz}$ that can be tolerated by network and storage limitations. This necessitates a system which selects 1 in 10 million interactions in a way which maximises the retention of the potentially interesting events. The events of interest occur at rates of, for example, $0.1 \mathrm{~Hz}$ for a Standard Model Higgs boson with a mass of $100 \mathrm{GeV}$; $500 \mathrm{kHz}$ for $\mathrm{W}$ production; $80 \mathrm{kHz}$ for $\mathrm{Z}$ production. This competes with inelastic backgrounds occuring at $1 \mathrm{GHz}$ and generic jets at $1 \mathrm{kHz}$. To achieve the reduction while retaining events of interest, a three tier trigger system has been devised. This system has been exercised by cosmic ray events in 2008/9 and single LHC beam delivery in 2008.

\section{Trigger Architecture and Trigger Menu at $\mathscr{L}=10^{31} \mathrm{~cm}^{-2} \mathrm{~s}^{-1}$}

At Level 1 (L1), coarse granularity muon or calorimeter objects are constructed in synch with the LHC bunch crossing rate of $40 \mathrm{MHz}$. The calorimeter objects are formed using the liquid argon and tile calorimeters [1] based on towers of size $0.1 \times 0.1$ in $\eta$ and $\phi$ and enable a determination of the transverse energy so that electron, photon, jet, missing Et, sum Et and tau triggers are defined. Isolation requirements may be imposed at L1 based on the activity in a cone surrounding a calorimeter object. Muon objects are formed in the Resistive Plate Chambers (RPC) which form the barrel section $(|\eta|<1.05)$ of the muon detector and Thin Gap Chambers (TGC) which form the endcap $(1.05<\eta<2.4)$. The trigger selects muon candidates based on their transverse momentum in regions of $\phi$ and $\eta$. Thresholds are applied to the L1 objects and a decision is made within 2.5 $\mu \mathrm{s}$ whether to retain the event. This selection reduces the rate which is passed into Level 2 (L2) to $75 \mathrm{kHz}$. The L1 muon or calorimeter object defines a region of interest (ROI) which is a cone around the $(\eta, \phi)$ position of the identified object so that at L2, identification algorithms for muons, calorimeter objects and tracks operate only on the ROI supplied by L1. This is a significant saving in processing time since the ROI typically corresponds to $2 \%$ of the full event size. Thresholds are applied at L2 and the decision must be made on average within $40 \mathrm{~ms}$ whether to keep the event. This reduces the output rate to $3 \mathrm{kHz}$. The level 2 (L2) and third level "event filter" (EF) trigger are software triggers which run on commercial processors and utilise the full detector granularity. At present there are approximately 950 PC boxes installed for L2 and EF and many of these can be switched between L2 and EF usage. At the EF, the algorithms match those of the offline algorithms with only minor differences and the full event can be accessed. This results in a processing time for the EF of four seconds after which the target output rate of $200 \mathrm{~Hz}$ is achieved.

A set of triggers, known as the menu, has been defined for the expected instantaneous LHC luminosity of $\mathscr{L}=10^{31} \mathrm{~cm}^{-2} \mathrm{~s}^{-1}$ during the early data-taking. Each "chain" in this menu is composed of a L1, L2 and EF object and each has an associated prescale at each level which allows the rate to be limited by accepting only a fraction of the events which pass a particular trigger. Table 1 shows the expected rates at a centre of mass energy of $14 \mathrm{TeV}$ for the lowest momentum threshold electron, muon, tau, jet and missing energy triggers. Since the LHC will collide beams at an energy of $7 \mathrm{TeV}$ initally, the rates will be reassessed and the thresholds lowered. Table 1 shows examples of the lowest unprescaled threshold EF triggers. 
Table 1: Examples of trigger rates. Thresholds are in $\mathrm{GeV}$.

\begin{tabular}{|l|c|c|c|c|c|}
\hline L1 & E/ $\gamma 3$ & Muon 4 & Tau 6 & Missing Et 15 & Jet 10 \\
\hline Prescale & 60 & 1 & 750 & 30000 & 42000 \\
\hline Rate $(\mathrm{Hz})$ & 674 & 1730 & 19 & 2.5 & 4 \\
\hline \hline EF & E/ $\gamma 12$ & Muon 10 & Tau 60 & Jet 120 & \\
\hline Rate $(\mathrm{Hz})$ & 19 & 18 & 10 & 9 & \\
\hline
\end{tabular}
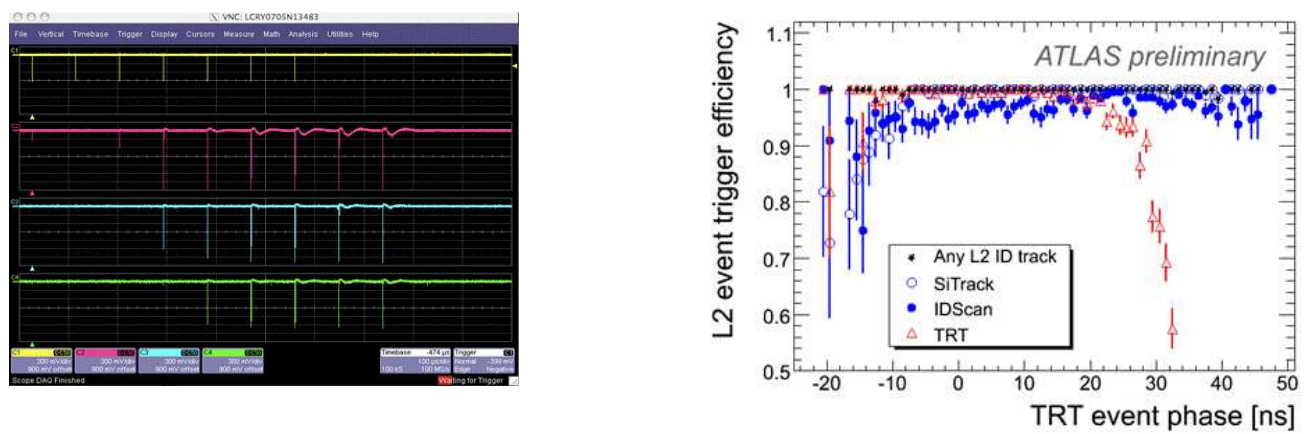

Figure 1: BPTX signal (yellow) with MBTS signals (pink, blue, green) show synchronised be-Figure 2: Tracking efficiency of each L2 tracking haviour during single beam running.

algorithm measured in cosmic data.

\section{Commissioning with Single LHC Beam Data}

In September 2008, a single beam was circulated at the injection energy of $450 \mathrm{GeV}$ in the LHC ring. A beam collimator was placed into the beam trajectory, giving rise to a spray of particles entering the ATLAS detector. This produced startling images of the detector experiencing high occupancy. During this phase, the mechanisms by which ATLAS detects the beam were deployed. During collisions, it is vital that these mechanisms are synchronised with the trigger system in order for it to capture events. The electrostatic beam pickup (BPTX) [2] device is mounted on the beampipe adjacent to ATLAS and gives a signal when a bunch is detected. The Minimum Bias Trigger Scintillators (MBTS) are mounted on the inner surface of the liquid argon endcap crysotats at $2.12<|\eta|<2.85$ and each one should be in synch with the bunches. Figure 1 shows their performance. Subsequent synchronisation of each trigger system with these devices will be critical.

\section{Commissioning with Cosmic Ray Data}

Nature has provided a steady supply of commissioning data in the form of cosmic ray muons. Approximately 270 million cosmic ray events were collected in 2008/9. An altered trigger menu is used during cosmic ray data-taking. The menu is based on the $\mathscr{L}=10^{31} \mathrm{~cm}^{-2} \mathrm{~s}^{-1}$ menu with some modifications. The high level trigger is generally not deployed in this phase except for the purpose of sending events into appropriate streams of data for processing. The track requirements were loosened so that the sample of tracks could be increased to enable detailed commissioning of the inner detector and alignment system. Each of the L2 tracking algorithms was tested and its efficiency measured with respect to the offline track finding (see figure 2). This illustrated a high efficiency for all algorithms and also highlighted problems, an example of which is the Transition Radiation Tracker [3] tracking algorithm which was not properly synchronised with the rest of the 


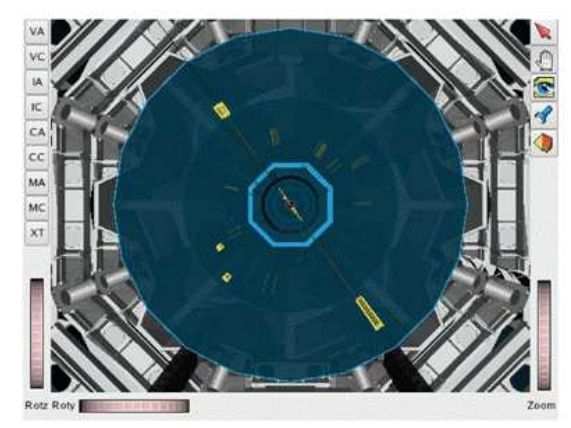

Figure 3: Tau signature in cosmic data.

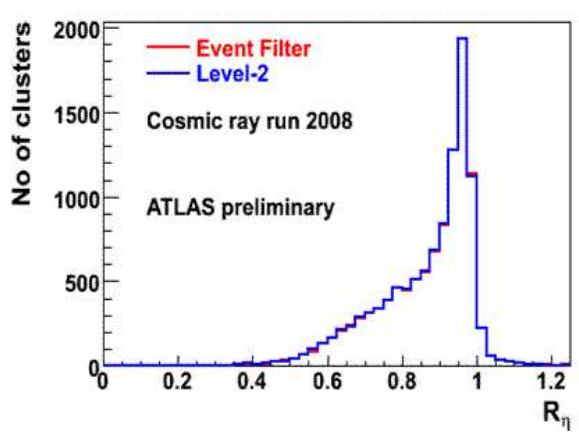

Figure 4: Shower shape variable in cosmic data.

L2 trigger. The EF tracking efficiency was also measured as a function of the impact parameter for the track with the magnetic field switched on and off.

Through this phase, operational procedures were established. The triggers were exercised at appreciable rates and the monitoring software underwent improvements throughout this period. While cosmic rays are muons, most of the other trigger objects at ATLAS also gathered events: figure 3 shows an event which passed the tau trigger which required deposits in the electromagnetic and hadronic calorimeter and a track is present in the inner detector. Figure 4 shows the distribution of a shower shape variable which is used to distinguish electrons and photons in the calorimeter. This displays agreement between L2 and EF.

\section{Plans for Collisions}

Collisions are awaited and it will take several steps to move from cosmic data-taking to the full trigger menu. During the first beam delivery, the BPTX will be required to signify the presence of beam. Next, the clocks in each individual trigger system will be synchronised with the LHC bunch group structure. Here several configurations are possible and each will define a different commissioning configuration: 1) both bunches filled (collisions); 2) both empty (default cosmic configuration); 3) one side filled; 4) empty bunch crossing after filled bunches (useful for measuring the effect of pile-up). Following the clock commissioning, the L1 calorimeter calibrations will be performed so that the correct noise suppression levels are deployed. The HLT will be used at first only for streaming the data for processing while the HLT algorithms are run offline on the data for debugging purposes. The HLT will then be added one piece at a time and at first only recording the trigger decision but not rejecting events. When the algorithms are validated online, it will be possible to put the HLT to full use.

\section{References}

[1] ATLAS Collaboration, Detector and Physics Technical Design Report, CERN/LHCC/99-14 (1999)

[2] T. Pauly and C. Ohm, Proceedings of TIPP09, arXiv:0905.3648

[3] ATLAS Inner Detector Technical Design Report,CERN/LHCC/97-16 (1997) 